\title{
Epidemiologia molecolare di ceppi di Stenotrophomonas maltophilia isolati da pazienti con fibrosi cistica
}

\author{
Ersilia Fiscarelli', Giordano Dicuonzo², Giovanni Gherardi² \\ 'Laboratorio di Microbiologia, Ospedale Pediatrico Bambin Gesù, Roma \\ ${ }^{2}$ Dipartimento di Patologia Clinica e Microbiologia, Università Campus Bio-Medico, Roma.
}

Key words: Stenotrophomonas maltophilia, cystic fibrosis, PFGE, antimicrobial resistance, epidemiology

Molecular epidemiology of Stenotrophomonas maltophilia isolates from patients with cystic fibrosis

\section{SUMMARY}

Eighty-nine Stenotrophomonas maltophilia isolates were recovered from 42 cystic fibrosis patients in a paediatric hospital of Rome, Italy, during a I7-months period. Twenty patients presented repeated episodes of S. maltophilia isolates, with 2 to7 isolates per patient. The antimicrobial susceptibilities of the strains showed that trimethoprim-sulfamethoxazole was the most active antibiotic, inhibiting more than $90 \%$ of the strains, followed by levofloxacin, with 62 out of 89 isolates being sensitive (69.7\%). Genetic relatedness of S. maltophilia isolates was investigated by pulsed-field gel electrophoresis (PFGE). A total of 49 well-defined distinct Xbal PFGE profiles were identified, with 39 different PFGE types encountered. Twenty-one PFGE types were represented by multiple isolates, with the most frequent being PFGE type 4 (I I isolates), PFGE type 9 and II (7 isolates each), and PFGE type 3 (5 isolates). Persistence of S. maltophilia infection or colonization was identified in 17 patients with repeated episodes, while genetically distinct S. maltophilia isolates from the same patient was found in 6 cases. Genetically identical or highly related isolates from different patients were observed within 8 multiple strain PFGE types. This study revealed a high genetic heterogeneity among S. maltophilia isolates recovered from cystic fibrosis patients. All together these data seem to confirm the wide environmental distribution of this pathogen. Different items of acquisition were involved both outside and inside the nosocomial settings, with the crosstransmission observed among clinical isolates of S. maltophilia among different patients probably due by multiple independent acquisitions from the environment as a main mode of transmission.

\section{INTRODUZIONE}

Stenotrophomonas maltophilia è un bacillo Gram negativo non fermentante, ubiquitario, che si ritrova nelle piante, acqua, suolo, ed anche in ambienti nosocomiali $(6,19)$. Sebbene venga considerato un organismo a limitata patogenicità, recentemente sta assumendo sempre più importanza quale patogeno nosocomiale, particolarmente nei pazienti immunocompromessi $(5,13)$. In questo gruppo di pazienti sono stati considerati diversi fattori di rischio che contribuiscono all'acquisizione di tale germe come agente infettivo o colonizzante a livello dell'apparato respiratorio, tra i quali ricordiamo il trattamento con antibiotici ad ampio spettro e prolungato nel tempo, lunga ospedalizzazione, ricovero nelle unità di Terapia Intensiva, ventilazione meccanica, cateterizzazione, neutropenia $(5,13,18,19)$.

La fibrosi cistica $(\mathrm{FC})$ è una malattia genetica autosomica recessiva legata al gene CFTR che risiede sul cromosoma 7 (16). La malattia della FC è caratterizzata da cicli di infiammazione ed infezione che portano ad una perdita progressiva della funzione polmonare. Nell'ultima decade è stata riportata un'alta incidenza di isolati di $S$. maltophilia in pazienti FC con associazione tra colonizzazione e danno polmonare, sebbene il reale ruolo di tale patogeno nella patogenesi della FC sia ancora da determinare $(1,3,5,10,12,14)$. Nonostante $S$. maltophilia venga considerato un importante patogeno nosocomiale, ancora poco si conosce sull'epidemiologia di questa specie e sul grado di correlazione genetica tra diversi isolati (13). È stato riportato in letteratura l'isolamento di tale specie dai sistemi idraulici, rubinetterie ed acqua sia dentro che fuori l'ambiente nosocomiale $(7,8,18)$. Allo scopo di definire i rapporti di clonalità tra diversi isolati clinici di S. maltophilia sono state impiegate diverse tecniche di tipizzazione molecolare, la cui utilità è resa ancor più necessaria dalla bassa variabilità di specie riscontrata utilizzando i test di tipizzazione fenotipici (8, 11, 18, 20). Attualmente la tecnica di tipizzazione 
molecolare della Pulsed Field Gel Electrophoresis (PFGE) è ampiamente riconosciuta ed accettata come la metodica di tipizzazione più accurata ed affidabile per la caratterizzazione molecolare degli isolati di S. maltophilia (20).

Lo scopo del nostro lavoro è stato quello di caratterizzare isolati clinici di $S$. maltophilia provenienti da pazienti FC del centro della Fibrosi Cistica dell'Ospedale Pediatrico Bambin Gesù, Roma, mediante PFGE con macrorestrizione con l'enzima $X b a I$. Abbiamo determinato il profilo di antibiotico resistenza e le correlazioni genetiche di 89 isolati di $S$. maltophilia provenienti da 52 pazienti FC in un periodo di osservazione di 17 mesi.

\section{MATERIALI E METODI}

\section{Dati epidemiologici e ceppi batterici}

Nel periodo 1 Gennaio 2003 - 31 Maggio 2004, sono stati arruolati in follow-up 193 pazienti del Centro di Fibrosi Cistica dell'Ospedale Pediatrico Bambino Gesù di Roma. Di questi 193 pazienti, 42 pazienti (23 femmine e 19 maschi) erano stati trovati positivi con 89 colture positive per $S$. maltophilia. La mediana dell'età fra i pazienti con coltura positiva era di 15 anni, con intervallo da 1 a 33. Gli isolati di S. maltophilia provenivano tutti da campioni respiratori ed in particolare venivano da: espettorato ( $n=68$ isolati), tracheoaspirato $(n=6$ isolati), lavaggio broncoalveolare $(n=1$ isolato), tampone faringeo ( $n=14$ isolati). I ceppi erano stati seminati su Agar Columbia non selettivo supplementato con 5\% di sangue di montone (Becton Dickinson) e su agar selettivo McConkey ed incubati a $35^{\circ} \mathrm{C}$ per $18-24$ ore, con una estensione del periodo di incubazione fino a 48 ore per quei ceppi a lenta crescita. Gli isolati di S. maltophilia sono stati identificati mediante i test fenotipici convenzionali ed in particolare mediante il sistema automatizzato Vitek 2 (bioMérieux, Marcy l'Etoile, France) e mediante le gallerie API 20 NE (bioMérieux, Marcy l'Etoile, France) per la conferma delle identificazioni dubbie.

\section{Profilo di sensibilità antibiotica}

I test di sensibilità agli antibiotici sugli isolati di S. maltophilia sono stati determinati mediante metodi manuali su micropiastre con tecnica di microdiluizione in brodo. Gli antibiotici testati sono stati i seguenti: ticarcillina-acido clavulanico (TIC), ceftazidime (CAZ), levofloxacina (LEV), ciprofloxacina (CIP), gentamicina (GEN), trimethoprim-sulfametossazolo (SXT). Le micropiastre sono state incubate a $35^{\circ} \mathrm{C}$ in $\mathrm{O} 2$ per $20-24$ ore secondo le raccomandazioni del CLSI (originariamente NCCLS) (2).

\section{Analisi mediante PFGE e "cluster analysis"}

Il DNA per la macrorestrizione è stato preparato come precedentemente descritto $(8,15,18)$ ed è stato sottoposto a digestione enzimatica con l'enzima $\mathrm{XbaI}$ per 20 ore a $37^{\circ} \mathrm{C}$. I parametri elettroforetici sono stati i seguenti: tempo iniziale e finale rispettivamente di $5 \mathrm{sec}$ and $35 \mathrm{sec}$, frammenti fatti correre per 20 ore ad una temperatura di $12^{\circ} \mathrm{C}$, un voltaggio di $6.0 \mathrm{~V} / \mathrm{cm}$ ed un angolo incluso di $120^{\circ} \mathrm{C}$. Due isolati con profili di PFGE che avevano fino a tre bande di differenza sono stati considerati geneticamente correlati e facenti parte dello stesso tipo di PFGE. Isolati con 4 o più bande di differenza sono stati considerati geneticamente non correlati e dunque facenti parte di gruppi clonali distinti (diversi tipi di PFGE). I tipi di PFGE sono stati analizzati con Bionumerics (versione 2.5; Applied Maths, Ghent, Belgium), normalizzando i profili di PFGE con lo standard. Il confronto dei profili è stato eseguito mediante il metodo UPGMA ("unweighted pair group method with arithmetic averages") utilizzando il coefficiente di similarità di Dice ed una tolleranza nella posizione delle bande del $1.5 \%$.

\section{RISULTATI}

Nel nostro studio condotto su pazienti del Centro della Fibrosi Cistica dell'ospedale Pediatrico Bambino Gesù di Roma sono stati isolati da 42 pazienti FC, 89 isolati di S. maltophilia durante un periodo di 17 mesi. Ventidue pazienti (52\%) presentavano un singolo episodio di infezione o colonizzazione da S. maltophilia, i rimanenti 20 pazienti $(48 \%)$ presentavano episodi ripetuti (tabella 1). Tra i 20 pazienti con episodi ripetuti di infezione o colonizzazione da S. maltophilia, 12 pazienti presentavano 2 isolati, da 4 pazienti ne venivano isolati 3 , da 2 pazienti ne venivano isolati 4 , da un paziente ne venivano isolati 5 , da un altro paziente 6 , ed infine in 2 pazienti si riscontravano 7 isolati (tabella 1). Il picco di età di acquisizione d' infezione o colonizzazione da $S$. maltophilia tra i pazienti con episodi singoli di isolati variava da 6 a 10 anni; tra i pazienti con episodi ripetuti, il picco variava tra 11 e 15 anni. S. maltophilia veniva isolato come singolo organismo in 19 casi su 89 campioni polmonari $(21 \%)$, in combinazione con altri organismi in 70 casi $(79 \%)$ (dati non mostrati).

I risultati dei test di sensibilità agli antibiotici per gli isolati di $S$. maltophilia sono illustrati in tabella 1. Il SXT si è dimostrato l'antibiotico più attivo inibendo più del $90 \%$ dei ceppi, seguito dalla LVX attiva sul $70 \%$ dei ceppi, TIC attiva sul 30\% dei ceppi CAZ $(22 \%$ di ceppi sensibili), e CIP (20\%). Soltanto il 6\% dei ceppi si mostrava sensibile alla gentamicina (GEN).

Tra gli 89 isolati di $S$. maltophilia genotipizzati mediante PFGE sono stati trovati 49 distinti profili di PFGE, con precisamente 39 tipi di PFGE e 
10 sottotipi, con una percentuale di similarità che variava tra il $66 \%$ ed il 100\% (figura I, tabella 1). I profili di PFGE trovati in isolati geneticamente correlati, facenti parte cioè dello stesso gruppo clonale, mostravano percentuale di similarità $\geq 90 \%$ e differivano di non più di 3 bande (figura I). Come illustrato in tabella 1,17 dei 22 pazienti cronicamente infettati o colonizzati da S. maltophilia mostravano isolati geneticamente identici o correlati (figura I, tabella 1). In totale, 21 tipi di PFGE erano costituiti da più di un isolato e 18 tipi di PFGE erano costituiti da isolati singoli (figura I, tabella 1). Dei 21 tipi di PFGE costituiti da almeno due isolati, 6 tipi erano caratterizzati da diversi sottotipi di PFGE (gli isolati con diverso sottotipo, sono isolati appartenenti allo stesso tipo di PFGE, che mostrano alla PFGE profili correlati, ma non identici) (figura I). Più frequentemente è stato riscontrato il PFGE di tipo 4 (11 isolati), seguito dal tipo 9 (7 isolati), tipo 11 (7 isolati), e tipo 3 (5 isolati) (figura I, tabella 1).

In 6 pazienti con episodi ripetuti di infezione o colonizzazione sono stati trovati isolati di S. maltophilia geneticamente diversi, facenti parte di diversi gruppi clonali $(\mathrm{B}, \mathrm{F}, \mathrm{N}, \mathrm{S}, \mathrm{AG}$, and $\mathrm{AJ})$ (figura I, tabella 1).

In 8 gruppi di PFGE sui 21 rappresentati da isolati multipli sono stati osservati isolati di S. maltophilia geneticamente correlati provenienti da pazienti diversi (PFGE tipi 1, 2, 4, 5, 7, 11, e 12) (figura I, tabella 1). Il principale gruppo caratterizzato da isolati di $S$. maltophilia provenienti da più di un paziente era rappresentato dal gruppo di PFGE 4, caratterizzato da 11 isolati provenienti da 4 pazienti diversi (figura I, tabella 1).

\section{DISCUSSIONE}

S. maltophilia è considerato un microrganismo a bassa patogenicità, che tuttavia sta divenendo sempre più importante nelle infezioni nosocomiali, soprattutto nei pazienti immunocompromessi. Questo microrganismo viene sempre più di frequente isolato come agente patogeno dal tratto respiratorio e dal tratto urinario, ed è una causa importante di batteremia secondaria (6).

In questo studio sono stati caratterizzati 89 isolati di S. maltophilia provenienti da 42 pazienti con Fibrosi Cistica. Il picco di età di acquisizione dell'infezione o colonizzazione da $S$. maltophilia corrispondeva con quello trovato in uno studio precedente di Valdezate at al. (18), dove in un periodo di 8 anni è stato trovato un picco di età di acquisizione di 6-10 anni tra i pazienti con episodi singoli da $S$. maltophilia, e di 16-20 tra i pazienti con episodi ripetuti.

I risultati di antibiotico resistenza per ceppi di $S$. maltophilia studiati erano in accordo con quelli descritti in letteratura $(9,17)$. In particolare l'antibiotico SXT è risultato il più attivo tra tutti quelli testati con una percentuale di ceppi sensibili al SXT di più del $90 \%$. Tuttavia il fatto di non aver trovato nessun antibiotico attivo sul $100 \%$ dei ceppi di $S$. maltophilia suggerisce la necessità comunque di eseguire $i$ test di sensibilità agli antibiotici in vitro per questa specie batterica. Al contrario, l'alto tasso di antibiotico-resistenza riscontrato e l'avere osservato simili profili di antibiotico-resistenza rende tale metodica fenotipica poco affidabile come metodo di tipizzazione per tale specie.

In 6 casi è stata osservata eterogeneità tra isolati di S. maltophilia provenienti dallo stesso paziente. Tale eterogeneità potrebbe essere dovuta all'acquisizione di tal ceppi da diverse fonti ambientali, probabilmente fuori dall'ambiente ospedaliero. Tale osservazione potrebbe essere suggerita dal fatto che la quasi totalità dei tipi di PFGE riscontrati in questi 6 casi erano gruppi di PFGE unici.

Di notevole interesse è stato l'aver trovato isolati con profili di PFGE identici o altamente correlati provenienti da pazienti diversi. Tale fenomeno è stato possibile osservarlo in 8 casi (tabella 1 ). In particolare sono stati osservati isolati provenienti da pazienti diversi che hanno frequentato lo stesso reparto ma in giorni diversi. Questo potrebbe far supporre l'ambiente ospedaliero come la possibile sorgente principale, con acquisizioni multiple indipendenti nel tempo. Infatti, è stata descritta precedentemente in letteratura l'ampia distribuzione di tale specie batterica nell'ambiente ed in particolare è stato possibile isolare ceppi di $S$. maltophilia dai sistemi idraulici e da altra strumentazione in ambito ospedaliero $(7,8,11)$. Purtroppo, non è stato possibile eseguire campionamenti ambientali nel nostro centro e dunque non è stato possibile ottenere alcuna informazione sulle possibili sorgenti di acquisizione e sulle vie di trasmissione di $S$. maltophilia nel nostro ospedale.

Interessante è stato l'avere osservato isolati geneticamente identici di S. maltophilia provenienti da due fratelli (pazienti $\mathrm{C} 1$ e $\mathrm{C} 2$ ), il che farebbe supporre che in questo caso la sorgente ambientale per l'acquisizione di S. maltophilia sia fuori dall'ambiente ospedaliero, probabilmente nell'ambiente domestico. Non è comunque da escludere completamente la possibilità di una trasmissione tra soggetti. L'osservazione di isolati di S. maltophilia geneticamente correlati provenienti da due fratelli era stata già descritta precedentemente da Krewinski et al $(4,11)$.

In conclusione appare assolutamente necessario stabilire una correlazione epidemiologica fra diversi isolati clinici di $S$. maltophilia, dato che si potrebbero ottenere risultati inattesi. Da questo studio è emersa una alta variabilità genotipica tra 
gli isolati clinici di S. maltophilia provenienti da pazienti FC. Tale eterogeneità era dovuta non soltanto alla diversità genotipica tra diversi ceppi provenienti da pazienti diversi, ma anche da ceppi di S. maltophilia provenienti dallo stesso paziente, come è stato possibile rilevare in diversi casi. Sono stati osservati gruppi clonali di ceppi di $S$. maltophilia sia provenienti dallo stesso paziente, fenomeno che prende il nome di "persistenza" della infezione o colonizzazione da S. maltophilia, sia gruppi clonali di isolati provenienti da pazienti diversi. Complessivamente questi dati sembrerebbero confermare il coinvolgimento di diversi modi di acquisizione e trasmissione di ceppi di S. maltophilia, che riguarderebbero ambienti sia dentro che fuori l'ospedale. Risulta assolutamente necessario ottenere più informazioni sui "reservoirs" nosocomiali e sui modi di acquisizione di tale specie batterica per meglio comprendere l'epidemiologia di S. maltophilia e quindi improntare delle misure di controllo e sorveglianza efficaci.

Tabella I. Dati clinici, profili di antibiotico-resistenza e tipi di PFGE osservati tra gli 89 isolati di S. maltophilia studiati

\begin{tabular}{|c|c|c|c|c|c|c|}
\hline Paziente & $\begin{array}{l}\text { Età } \\
\text { (sesso) }\end{array}$ & $\begin{array}{l}\text { Sito } \\
\text { corporeo }\end{array}$ & Reparto & $\begin{array}{c}\mathrm{n} . \\
\text { isolati }\end{array}$ & $\begin{array}{l}\text { Tipi di PFGE } \\
\text { (n. isolati) }\end{array}$ & $\begin{array}{l}\text { Profili di antibiotico-resistenza } \\
\text { (n. isolati) }\end{array}$ \\
\hline $\bar{A}$ & 19(M) & ESP & $\mathrm{DH}$ & I & $\mathrm{I} . \mathrm{I}$ & LVX,SXT \\
\hline$\overline{\mathbf{B}}$ & $16(M)$ & ESP & DH & 6 & $I . I(I), 6 . I(4), I 4 . I(I)$ & SXT \\
\hline$\overline{\mathrm{T}}$ & $9(\mathrm{M})$ & ESP & $\mathrm{DH}$ & I & 14.1 & LVX,SXT \\
\hline$\overline{\mathrm{Cl}}$ & $16(\mathrm{M})$ & ESP & $\mathrm{MI}$ & $\mathrm{I}$ & 2.1 & LVX,SXT \\
\hline $\mathrm{C} 2$ & I3(M) & ESP & $\mathrm{MI}$ & I & 2.1 & LVX,SXT \\
\hline D & 18(F) & ESP & $\mathbf{E}$ & 5 & 3.1 & CAZ, SXT \\
\hline $\bar{E}$ & $9(\mathrm{~F})$ & faringe & $\mathrm{DH}$ & I & 4.1 & TIC,CAZ,CIP,LVX,SXT \\
\hline$\overline{\mathbf{F}}$ & 6(M) & ESP & DH & 2 & $4.2,31.1$ & LVX,SXT \\
\hline $\mathbf{G}$ & I5(M) & ESP & DH & 7 & $4 . I(I), 4.2(6)$ & TIC,LVX,SXT \\
\hline$\overline{\mathbf{A D}}$ & $2(M)$ & at & DH & 2 & 4.3 & SXT \\
\hline $\mathrm{H}$ & $10(\mathrm{~F})$ & bal & UTI & I & 5.1 & TIC,CAZ,GEN,CIP,LVX,SXT \\
\hline I & $15(\mathrm{~F})$ & ESP & $\mathrm{MI}$ & $\mathrm{I}$ & 5.1 & TIC,CAZ,GEN,CIP,LVX,SXT \\
\hline J & $18(\mathrm{~F})$ & faringe & $\mathrm{DH}$ & I & 7.1 & TIC,CAZ,CIP,LVX,SXT \\
\hline $\mathbf{K}$ & $I I(M)$ & ESP & DH & 2 & 7.2 & TIC \\
\hline $\mathbf{L}$ & I4(F) & faringe & $\mathbf{D H}$ & 2 & 8.1 & LVX,SXT \\
\hline$M$ & 20(F) & ESP & DH & 7 & $9.1(5), 9.2(2)$ & SXT \\
\hline $\mathbf{N}$ & $26(M)$ & ESP & MI & 4 & $10.1(3), 30.1(1)$ & LVX,SXT \\
\hline O & $I I(M)$ & ESP & MI & 3 & II.I(2), II.4(I) & TIC,CAZ,LVX,SXT \\
\hline$\overline{\mathbf{P}}$ & I4(F) & ESP & MI & 4 & II.2(3), I I.3(I) & LVX,SXT \\
\hline $\mathbf{Q}$ & 24(M) & ESP & MI & 2 & $12.1,12.2$ & LVX,SXT \\
\hline$\vec{R}$ & $16(F)$ & ESP & $\mathrm{DH}$ & I & 12.3 & GEN \\
\hline$\underline{\mathbf{S}}$ & 4(F) & ESP & DH & 3 & $13.1(2), 34.1(1)$ & LVX,SXT(2); SXT(I) \\
\hline $\bar{U}$ & $22(M)$ & ESP & $\mathrm{MI}$ & I & 15.1 & CAZ,SXT \\
\hline$\overline{\mathrm{V}}$ & $\mathrm{I}(\mathrm{F})$ & at & $\mathrm{DH}$ & $\mathrm{I}$ & 16.1 & CIP,LVX,SXT \\
\hline$\underline{\mathbf{X}}$ & $26(M)$ & ESP & $\mathbf{D H}$ & 2 & I7.I & LVX,SXT \\
\hline $\mathbf{Y}$ & 10(F) & faringe & DH & 2 & I8.I & LVX,SXT \\
\hline$\overline{\mathbf{Z}}$ & $8(F)$ & faringe & DH & 2 & 19.1 & LVX,SXT \\
\hline$\overline{\mathrm{AA}}$ & $2(\mathrm{~F})$ & faringe & $\mathrm{P}$ & $\mathrm{I}$ & 20.1 & LVX,SXT \\
\hline $\mathrm{AB}$ & I3(F) & faringe & MI & $\mathrm{I}$ & 21.1 & TIC,LVX \\
\hline$\overline{A C}$ & $24(\mathrm{~F})$ & ESP & $\mathrm{DH}$ & $\mathrm{I}$ & 22.1 & TIC,CAZ,CIP,LVX,SXT \\
\hline$\overline{\mathrm{AK}}$ & $2 \mathrm{I}(\mathrm{F})$ & ESP & $\mathrm{MI}$ & I & 23.1 & TIC,CIP,LVX,SXT \\
\hline$\overline{\mathrm{AE}}$ & I8(M) & ESP & $\mathrm{MI}$ & I & 24.1 & SXT \\
\hline AF & I7(F) & ESP & DH & 3 & 25.1 & LVX,SXT \\
\hline \multirow[t]{2}{*}{$\overline{\mathbf{A G}}$} & 28(M) & ESP & DH & 2 & $26.1,36.1$ & LVX,SXT(I) \\
\hline & & & & & & TIC,CAZ,GEN,CIP,LVX,SXT(I) \\
\hline$\overline{\mathrm{AH}}$ & I5(F) & ESP & $\mathrm{MI}$ & I & 27.1 & CIP,LVX,SXT \\
\hline $\mathbf{A l}$ & I(M) & at & DH & 3 & $28 . I(1), 28.2(2)$ & TIC,CAZ,CIP,LVX,SXT \\
\hline$\overline{\mathbf{A J}}$ & I5(F) & faringe & DH & 2 & $29.1,32.1$ & TIC,LVX,SXT(I);R'(I) \\
\hline$\overline{\mathbf{A L}}$ & $3(F)$ & faringe & DH & 2 & 33.1 & SXT \\
\hline $\mathbf{A M}$ & $23(M)$ & ESP & DH & 2 & 35.1 & CIP,LVX \\
\hline $\mathrm{AN}$ & $12(\mathrm{~F})$ & ESP & $\mathrm{MI}$ & $\mathrm{I}$ & 37.1 & TIC,CAZ,CIP,LVX,SXT \\
\hline$\overline{\mathrm{AO}}$ & $27(\mathrm{~F})$ & ESP & $\mathrm{MI}$ & $\mathrm{I}$ & 38.1 & CIP,LVX,SXT \\
\hline $\mathrm{AP}$ & $33(\mathrm{M})$ & ESP & $\mathrm{DH}$ & I & 39.1 & CIP,LVX,SXT \\
\hline
\end{tabular}

Abbreviazioni. M: maschio; F: femmina; ESP: espettorato; bal: lavaggio bronchiale; at: aspirato tracheale; DH: Day Hospital;

E: Epatologia; P: Pneumologia; UTI: Unità terapia Intensiva; MI: Medicina Interna

${ }^{a}$ Antibiotici ai quali gli isolati sono risultati sensibili in base ai criteri del CLSI/NCCLS (2). TIC: ticarcillina-acido clavulanico; CAZ: ceftazidime; GEN: gentamicina; CIP: ciprofloxacina; LVX: levofloxacina; SXT: trimethoprim-sulfametossazolo.

b Un isolato è stato trovato resistente a tutti gli antibiotici testati.

In neretto sono stati indicati i pazienti con episodi ripetuti di S. maltophilia. 


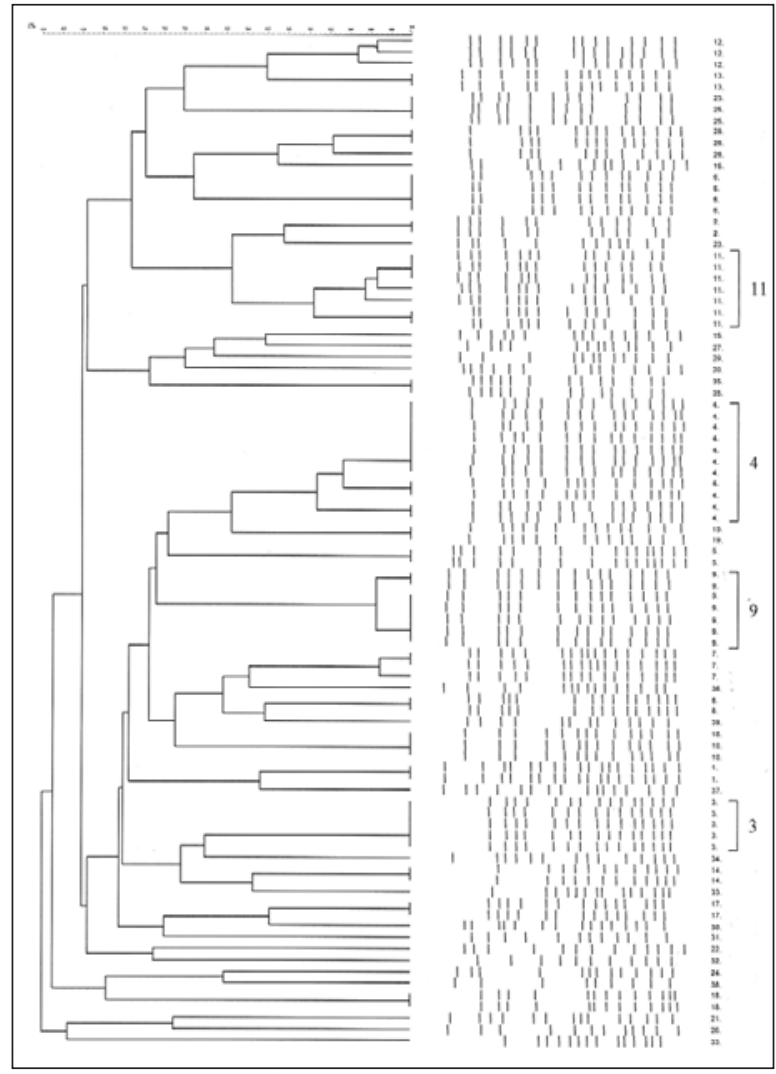

Figura I. Analisi filogenetica dei profili di PFGE degli 89 isolati di S. maltophilia studiati. Sono indicati sulla sinistra i sottotipi di PFGE con i 4 principali gruppi di PFGE trovati.

\section{BIBLIOGRAFIA}

1. Canton R, Valdezate S, Vindel A, Sanchez Del Saz B, Maiz L, Baquero F. Antimicrobial susceptibility profile of molecular typed cystic fibrosis Stenotrophomonas maltophilia isolates and differences with noncystic fibrosis isolates. Pediatr Pulmonol 2003; 35: 99-107.

2. Clinical and Laboratory Standards Institute/NCCLS. Performance standards for antimicrobial susceptibility testing; fifteenth informational supplement. CLSI/NCCLS document M100-S15, p. 40-42. Clinical and Laboratory Standards Institute, Wayne, Pa. USA 2005

3. da Silva Filho LVF, Tateno AF, Villoso LF, et al. Identification of Pseudomonas aeruginosa, Burkholderia cepacia complex, and Stenotrophomonas maltophilia in respiratory samples from cystic fibrosis patients using multiplex PCR. Pediatr Pulmonol 2004; 37: 537-47.

4. Demk CA, Stern RC, Doershuk CF. Stenotrophomonas maltophilia in cystic fibrosis: incidence and prevalence. Pediatr Pulmonol 1998; 25: 304-8.

5. Denton M. Stenotrophomonas maltophilia: an emerging problem in cystic fibrosis patients. Rev Med Microbiol 1997; 8: 15-9.

6. Denton M, Kerr KG. Microbiological and clinical aspects of infection associated with Stenotrophomonas maltophilia. Clin Microbiol Rev 1998; 11: 57-80.

7. Denton M, Kerr KG. Molecular epidemiology of Stenotrophomonas maltophilia isolated from cystic fibrosis patients. J Clin Microbiol 2002; 40: 1884.

8. Denton M, Todd NJ, Kerr KG, Hawkey PM,
Littlewood JM. Molecular epidemiology of Stenotrophomonas maltophilia isolated from clinical specimens from patients with cystic fibrosis and associated environmental samples. J Clin Microbiol 1998; 36: 1953-8.

9. Fadda G, Spanu T, Ardito F, et al. Antimicrobial resistance among non-fermentative Gram-negative bacilli isolated from the respiratory tracts of Italian inpatients: a 3-year surveillance study by the Italian epidemiological survey. Int J Antimicrob Agents 2004; 23: 254-61.

10. Goss CH, Mayer-Hamblett N, Aitken ML, Rubenfeld GD, Ramsey BW. Association between Stenotrophomonas maltophilia and lung function in cystic fibrosis. Thorax 2004; 59: 955-9.

11. Krzewinski JW, Nguyen CD, Foster JM, Burns JL. Use of random amplified polymorphic DNA to examine epidemiology of Stenotrophomonas maltophilia and Achromobacter (Alcaligenes) xylosoxidans from patients with cystic fibrosis. J Clin Microbiol 2001; 39: 3597-602.

12. Pathmanathan A, Waterer GW. Significance of positive Stenotrophomonas maltophilia culture in acute respiratory tract infection. Eur Respir J 2005; 25: 911-4.

13. Senol E. Stenotrophomonas maltophilia: the significance and role as a nosocomial pathogen. J Hosp Infect 2004; 57: 1-7.

14. Steinkamp G, Wiedemann B, Rietschel E, et al. Prospective evaluation of emerging bacteria in cystic fibrosis. J Cyst Fibros 2005; 4: 41-8.

15. Tenover FC, Arbeit RD, Goering RV, et al. Interpreting chromosomal DNA restriction patterns produced by pulsed-field gel electrophoresis:criteria for bacterial strain typing. J Clin Microbiol 1995; 33: 2233-9.

16. Trezise AE, Szpirer C, Buchwald M. Location of the gene encoding the cystic fibrosis transmembrane conductance regulator (CFTR) in the rat to chromosome 4 and implications for the evolution of mammalian chromosomes. Genomics 1992; 14: 869-74.

17. Valdezate S, Vindel A, Loza E, Baquero F, Canton R. Antimicrobial susceptibilities of unique Stenotrophomonas maltophilia clinical strains. Antimicrob Agents Chemother 2001; 45: 1581-4.

18. Valdezate S, Vindel A, Maiz L, Baquero F, Escobar H, Canton R. Persistance and variabilità of Stenotrophomonas maltophilia in cystic fibrosis patients, Madrid, 1991-1998. Emerg Infect Dis 2001; 7: 113-22.

19. Valdezate S, Vindel A, Martin-Davila P, Sanchez Del Saz B, Baquero F, Canton R. High genetic diversity among Stenotrophomonas maltophilia strains despite their originating at a single hospital. J Clin Microbiol 2004; 42: 693-9.

20. Yao JDC, Conly JM, Krajden M. Molecular typing of Stenotrophomonas (Xanthomonas) maltophilia by DNA macrorestriction analysis and random amplified polymorphic DNA analysis. J Clin Microbiol 1995; 33: 2195-8.

\section{Giovanni Gherardi}

Dipartimento di Patologia Clinica e

Microbiologia

Università Campus Bio-Medico

Via Emilio Longoni 47-00155 Roma

Tel. 06 22541790; Fax 0622541791

E-mail: g.gherardi@unicampus.it 